\title{
Search for giant planets in M67
}

\section{Excess of hot Jupiters in dense open clusters ${ }^{\star}$}

\author{
A. Brucalassi ${ }^{1,2}$, L. Pasquini ${ }^{3}$, R. Saglia ${ }^{1,2}$, M. T. Ruiz ${ }^{4}$, P. Bonifacio ${ }^{5}$, I. Leão ${ }^{3,6}$, B. L. Canto Martins ${ }^{6}$, \\ J. R. de Medeiros ${ }^{6}$, L. R. Bedin ${ }^{7}$, K. Biazzo ${ }^{8}$, C. Melo ${ }^{9}$, C. Lovis ${ }^{10}$, and S. Randich ${ }^{11}$ \\ 1 Max-Planck für extraterrestrische Physik, 85741 Garching bei München, Germany \\ e-mail: abrucala@mpe.mpg.de \\ 2 University Observatory Munich, Ludwig Maximillian Universitaet, Scheinerstrasse 1, 81679 Munich, Germany \\ 3 ESO - European Southern Observatory, Karl-Schwarzschild-Strasse 2, 85748 Garching bei München, Germany \\ Astronomy Department, Universidad de Chile, 1058 Santiago, Chile \\ 5 GEPI, Observatoire de Paris, CNRS, Univ. Paris Diderot, Place Jules Janssen, 92190 Meudon, France \\ ${ }^{6}$ Universidade Federal do Rio Grande do Norte, 59300-000 Natal, Brazil \\ 7 Istituto Nazionale di Astrofisica, Osservatorio Astronomico di Padova, 35141 Padova, Italy \\ 8 Istituto Nazionale di Astrofisica, Osservatorio Astronomico di Catania, 78 Catania, Italy \\ 9 ESO - European Southern Observatory, 85748 Santiago, Chile \\ 10 Observatoire de Genève, 1290 Sauverny, Switzerland \\ 11 Istituto Nazionale di Astrofisica, Osservatorio Astrofisico di Arcetri, 20125 Firenze, Italy \\ Received 14 October 2015 / Accepted 26 May 2016
}

\begin{abstract}
Since 2008 we used high-precision radial velocity (RV) measurements obtained with different telescopes to detect signatures of massive planets around main-sequence and evolved stars of the open cluster (OC) M67. We aimed to perform a long-term study on giant planet formation in open clusters and determine how this formation depends on stellar mass and chemical composition. A new hot Jupiter (HJ) around the main-sequence star YBP401 is reported in this work. An update of the RV measurements for the two HJ host-stars YBP1194 and YBP1514 is also discussed. Our sample of 66 main-sequence and turnoff stars includes 3 HJs, which indicates a high rate of HJs in this cluster $\left(5.6_{-2 .}^{+5.4} \%\right.$ for single stars and $4.5 \%{ }_{-25}^{+4.5} \%$ for the full sample). This rate is much higher than what has been discovered in the field, either with RV surveys or by transits. High metallicity is not a cause for the excess of HJs in M67, nor can the excess be attributed to high stellar masses. When combining this rate with the non-zero eccentricity of the orbits, our results are qualitatively consistent with a $\mathrm{HJ}$ formation scenario dominated by strong encounters with other stars or binary companions and subsequent planet-planet scattering, as predicted by $N$-body simulations.
\end{abstract}

Key words. planets and satellites: gaseous planets - techniques: radial velocities

\section{Introduction}

Hot Jupiters (HJs) are defined as giant planets $\left(M_{\mathrm{p}}>0.3 M_{\mathrm{Jup}}\right)$ on short-period orbits $(P<10$ days $)$. They show an occurrance rate of $\sim 1.2 \%$ around Sun-like field stars (Wright et al. 2012; Mayor et al. 2011). These close-in giant planets are highly unlikely to have formed in situ, and it is believed that they form beyond the snow line where solid ices are more abundant, allowing the planet cores to grow several times more massive than in the inner part of the proto-planetary disk before undergoing an inward migration. Of the mechanisms that are able to trigger migration, the two supported most often are dynamical interaction with the circumstellar disk (Goldreich \& Tremaine 1980; Lin \& Papaloizou 1986; Ward 1997, type II migration) and gravitational scattering caused by other planets (Rasio \& Ford 1996; Lin \& Ida 1997, planet-planet scattering). Other ideas include violent migration mechanism such as dynamical encounters with a third body (multi-body dynamical interaction). In particular, recent $N$-body simulations

* Based on observations collected at the ESO $3.6 \mathrm{~m}$ telescope (La Silla), at the $1.93 \mathrm{~m}$ telescope of the Observatoire de HauteProvence (OHP), at the Hobby Eberly Telescope (HET), at the Telescopio Nazionale Galileo (TNG, La Palma) and at the Euler Swiss Telescope. have shown that a planetary system inside a crowded birthenvironment can be strongly destabilized by stellar encounters and dynamical interaction, which also favours the formation of HJs (Davies et al. 2014; Malmberg et al. 2011; Shara et al. 2016). Open clusters (OCs) hold great promise as laboratories in which properties of exoplanets and theories of planet formation and migration can be explored.

In Paper I (Pasquini et al. 2012) we described a radial velocity (RV) survey to detect the signature of giant planets around a sample of main-sequence (MS) and giant stars in M67. The first three planets discovered were presented in Brucalassi et al. (2014, Paper II). One goal of this project is to investigate whether and how planet formation is influenced by the environment. Recent planet search surveys in OCs support that the statistics in OCs is compatible with the field (Malavolta et al. 2016; Brucalassi et al. 2014; Meibom et al. 2013; Quinn et al. 2014, 2012). In this work we show that for M67 the frequency of HJs is even higher than in the field.

\section{Observations and orbital solutions}

Of the 88 stars in the original M67 sample, 12 have been found to be binaries (Pasquini et al. 2012). Two additional binaries have recently been discovered (Brucalassi et al. 2016). The final sample therefore comprises 74 single stars (53 MS and turnoff 
Table 1. Stellar parameters of the three M67 stars newly found to host planet candidates.

\begin{tabular}{lrrr}
\hline \hline Parameters & YBP401 & YBP1194 & YBP1514 \\
\hline$\alpha(\mathrm{J} 2000)$ & $08: 51: 19.05$ & $08: 51: 00.81$ & $08: 51: 00.77$ \\
$\delta(\mathrm{J} 2000)$ & $+11: 40: 15.80$ & $+11: 48: 52.76$ & $+11: 53: 11.51$ \\
Spec. type & $\mathrm{F} 9 \mathrm{~V}$ & $\mathrm{G} 5 \mathrm{~V}$ & $\mathrm{G} 5 \mathrm{~V}$ \\
$m_{V}[\mathrm{mag}]$ & $13.70^{a}$ & $14.6^{a}$ & $14.77^{a}$ \\
$B-V[\mathrm{mag}]$ & $0.607^{a}$ & $0.626^{a}$ & $0.680^{a}$ \\
$M \star\left[M_{\odot}\right]$ & $1.14 \pm 0.02^{b}$ & $1.01 \pm 0.02^{b}$ & $0.96 \pm 0.01^{b}$ \\
$\log g[\mathrm{cgs}]$ & $4.30 \pm 0.035^{d}$ & $4.44 \pm 0.05^{c}$ & $4.57 \pm 0.06^{d}$ \\
$T_{\text {eff }}[\mathrm{K}]$ & $6165 \pm 64^{d}$ & $5780 \pm 27^{c}$ & $5725 \pm 45^{d}$ \\
\hline
\end{tabular}

References. ${ }^{(a)}$ Yadav et al. (2008). ${ }^{(b)}$ Pietrinferni et al. (2004) and Girardi et al. (2000). ${ }^{(c)}$ Önehag et al. (2011). ${ }^{(d)}$ Pasquini et al. (2008) and Pace et al. (2012).

stars and 21 giants) that are all high-probability members (from proper motion and radial velocity) of the cluster according to Yadav et al. (2008) and Sanders (1977).

The star YBP401 shows significant indications of a HJ companion and is analysed here in detail. We also present an update of the RV measurements for the stars YBP1194 and YBP1514, for which two other HJs were announced in our previous work (Brucalassi et al. 2014).

Basic stellar parameters $\left(V, B-V, T_{\text {eff }}, \log g\right)$ with their uncertainties were taken from the literature. A distance modulus of $9.63 \pm 0.05$ (Pasquini et al. 2008) and a reddening of $E(B-V)=0.041 \pm 0.004$ (Taylor 2007) were assumed, stellar masses and radii were derived using the 4 Gyr theoretical isochrones from Pietrinferni et al. (2004) and Girardi et al. (2000). The parameters estimated from isochrone fitting agree within the errors with the values adopted from the literature. The main characteristics of the three host stars are listed in Table 1.

The RV measurements were carried out using the HARPS spectrograph (Mayor et al. 2003) at the ESO $3.6 \mathrm{~m}$ telescope in high-efficiency mode (with $R=90000$ and a spectral range of 378-691 nm), with the SOPHIE spectrograph (Bouchy \& Sophie Team 2006) at the OHP $1.93 \mathrm{~m}$ telescope in highefficiency mode (with $R=40000$ and a range of 387-694 nm), with the HRS spectrograph (Tull 1998) at the Hobby Eberly Telescope (with $R=60000$ and a range of 407.6-787.5 nm), and with the HARPS-N spectrograph at the TNG on La Palma of the Canary Islands (spectral range of 383-693 nm and $R=$ 115000 ). Additional RV data points for giant stars have been observed between 2003 and 2005 (Lovis \& Mayor 2007) with the CORALIE spectrograph at the $1.2 \mathrm{~m}$ Euler Swiss telescope.

HARPS, SOPHIE, and HARPS-N are provided with a similar automatic pipeline. The spectra are extracted from the detector images and cross-correlated with a numerical G2-type mask. Radial velocities are derived by fitting each resulting crosscorrelation function (CCF) with a Gaussian (Baranne et al. 1996; Pepe et al. 2002). For the HRS, the radial velocities were computed using a series of dedicated routines based on IRAF and by cross-correlating the spectra with a G2 star template (Cappetta et al. 2012). We used nightly observations of the RV standard star HD 32923 to correct all observations for each star to the zero point of HARPS (as explained in Pasquini et al. 2012) and to take into account any instrument instability or systematic velocity shifts between runs. An additional correction was applied to the SOPHIE data to consider the low signal-to-noise ratio $(\mathrm{S} / \mathrm{N})$ of the observations (Santerne et al. 2012, see Eq. (1)).

The RV measurements of our target stars were studied by computing the Lomb-Scargle periodogram (Scargle 1982; Horne \& Baliunas 1986) and by using a Levenberg-Marquardt

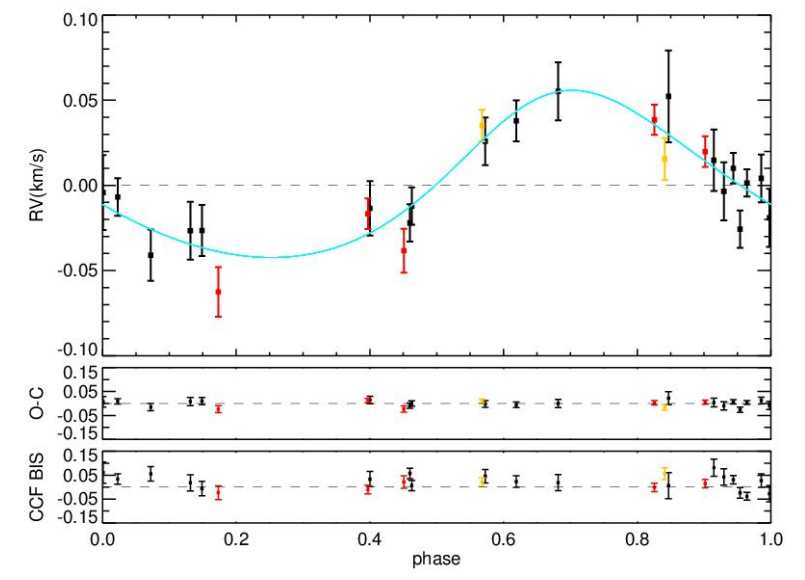

Fig. 1. Phased RV measurements and Keplerian best fit, best-fit residuals, and bisector variation for YBP401. Black dots: HARPS measurements, red dots: SOPHIE measurements, orange dots: HARPS-N measurements.

analysis (Wright \& Howard 2009, RVLIN) to fit Keplerian orbits to the radial velocity data. The orbital solutions were independently checked using the Yorbit program (Segransan et al. 2011) and a simple Markov chain Monte Carlo (MCMC) analysis (see Table A.2). We investigated the presence and variability of chromospheric active regions in these stars by measuring the variations of the core of the $\mathrm{H} \alpha$ line with respect to the continuum, following a method similar to the one described in Pasquini \& Pallavicini (1991). The more sensitive $\mathrm{Ca}$ II $\mathrm{H}$ and $\mathrm{K}$ lines were not accessible because of the low $\mathrm{S} / \mathrm{N}$ of our observations. For each case we verified the correlation between the RVs and the bisector span of the CCF (calculated following Queloz et al. 2001) or with the full width at half maximum (FWHM) of the CCF.

YBP401. According to Yadav et al. (2008), this F9V MS star has a membership probability of $97 \%$ and a proper motion shorter than 6 mas/yr with respect to the average. Vereshchagin et al. (2014) revised the membership list of Yadav et al. (2008) and expressed doubts about the cluster membership for YBP401. However, the RV value considered for YBP401 in Vereshchagin et al. (2014) has an uncertainty of $\sigma=130 \mathrm{~m} \mathrm{~s}^{-1}$ and is not consistent with our measurements by more than $1 \sigma$. Recently, Geller et al. (2015) confirmed YBP401 as a single cluster member.

This target has been observed since January 2008: $19 \mathrm{RV}$ points have been obtained with HARPS with a typical S/N of 15 (per pixel at $550 \mathrm{~nm}$ ) and a mean measurement uncertainty of $15 \mathrm{~m} \mathrm{~s}^{-1}$ including calibration errors. Five additional RV measurements were obtained with SOPHIE and two with HARPS-N, with measurement uncertainties of $9.0 \mathrm{~m} \mathrm{~s}^{-1}$ and $11.0 \mathrm{~m} \mathrm{~s}^{-1}$, respectively. The final $26 \mathrm{RV}$ measurements of YBP401 show a variability of $\sim 35 \mathrm{~m} \mathrm{~s}^{-1}$ and an average uncertainty of $\sim 14 \mathrm{~m} \mathrm{~s}^{-1}$ for the individual RV values. A clear peak is present in the periodogram (see Fig. A.1) at 4.08 days. A Keplerian orbit was adjusted to the RV data of YBP401 (see Fig. 1), and the resulting orbital parameters for the planet candidate are reported in Tables 2 and A.2. We note that the non-zero eccentricity is consistent with $e=0$ within $2 \sigma$ and the other parameters change by less than $1 \sigma$ when fixing $e=0$. We included the eccentricity in the data analysis, which resulted in a better fit $\left(\chi_{\text {red }}^{2} \sim 1\right)$ and in reduced RV residuals. However, more precise observations are needed to constrain small non-zero eccentricities and to avoid overinterpreting the results (see discussions in 
Table 2. Orbital parameters of the planetary candidates.

\begin{tabular}{lrrr}
\hline \hline Parameters & YBP401 & YBP1194 & YBP1514 \\
\hline$P$ [days] & $4.087 \pm 0.007$ & $6.959 \pm 0.001$ & $5.118 \pm 0.001$ \\
$T[\mathrm{JD}]$ & $2455974.3 \pm 0.5$ & $2455289.98 \pm 0.51$ & $2455986.3 \pm 0.3$ \\
$e$ & $0.15 \pm 0.08$ & $0.30 \pm 0.08$ & $0.28 \pm 0.09$ \\
$\omega[\mathrm{deg}]$ & $330.17 \pm 25.68$ & $99.36 \pm 20.47$ & $327.33 \pm 16.09$ \\
$K\left[\mathrm{~m} \mathrm{~s}^{-1}\right]$ & $49.06 \pm 3.50$ & $35.80 \pm 3.81$ & $50.06 \pm 5.03$ \\
$m \sin i\left[M_{\text {Jup }}\right]$ & $0.46 \pm 0.05$ & $0.32 \pm 0.03$ & $0.40 \pm 0.38$ \\
$\gamma\left[\mathrm{km} \mathrm{s}^{-1}\right]$ & $33.178 \pm 0.006$ & $34.185 \pm 0.002$ & $34.058 \pm 0.003$ \\
$\sigma(\mathrm{O}-\mathrm{C})\left[\mathrm{m} \mathrm{s}^{-1}\right]$ & 12.74 & 12.28 & 14.43 \\
\hline
\end{tabular}

Notes. $P$ : period, $T$ : time at periastron passage, $e$ : eccentricity, $\omega:$ argument of periastron, $K$ : semi-amplitude of the RV curve, $m \sin i$ : planetary minimum mass, $\gamma$ : average radial velocity, $\sigma(\mathrm{O}-\mathrm{C})$ : dispersion of Keplerian fit residuals.

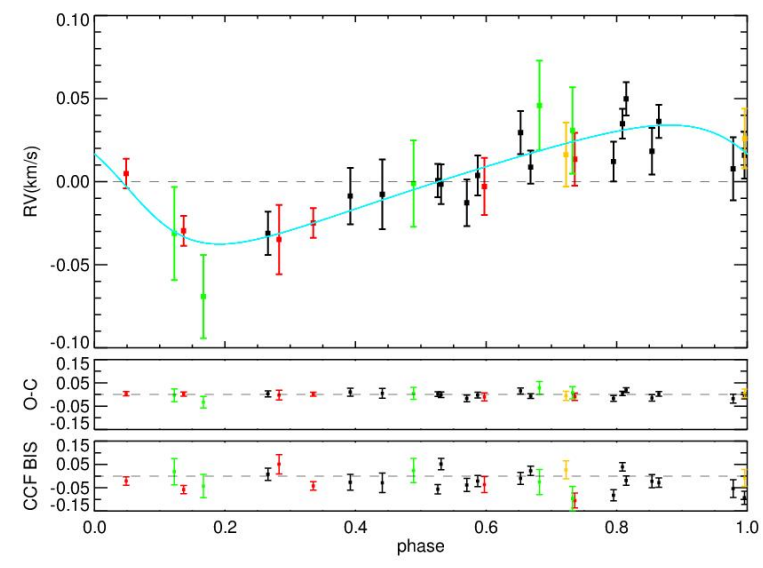

Fig. 2. Phased RV measurements and Keplerian best fit, best-fit residuals, and bisector variation for YBP1194. Same symbols as in Fig. 1, green dots: HRS measurements.

Zakamska et al. 2011; Pont et al. 2011). The residuals have an rms amplitude of $\sim 13 \mathrm{~m} \mathrm{~s}^{-1}$ and the periodogram of the residuals does not show any clear periodicity when the main signal is removed (see Fig. A.1). Neither the bisector span nor the activity index present correlations with the RV variations (see Fig. A.2); this excludes activity-induced variations of the shape or the spectral lines as the source of the RV measurements.

YBP1194 and YBP1514. We have now collected 29 measurements for both YBP1194 and YBP1514, spanning seven years. The average RV uncertainty is $\sim 13.0 \mathrm{~m} \mathrm{~s}^{-1}$ for HARPS and SOPHIE, $\sim 26.0 \mathrm{~m} \mathrm{~s}^{-1}$ for HRS and $\sim 8.0 \mathrm{~m} \mathrm{~s}^{-1}$ for HARPS-N. Figures 2 and 3 show the phase-folded data points together with the best-fit solution and the residual over the time. The peak in the periodogram is more pronounced and the RV signal is better determined (see Fig. A.1) than in Brucalassi et al. (2014). When the planet signature is removed, the rms of the residuals is $\sim 12.3 \mathrm{~m} \mathrm{~s}^{-1}$ for YBP1194 and $\sim 14.4 \mathrm{~m} \mathrm{~s}^{-1}$ for YBP1514.

We note that the resulting updated orbital parameters are consistent within the errors with the previously published data (see Tables 2 and A.2).

\section{Frequency of hot Jupiters in OCs}

The most striking result is that with the star YBP401 we have found three HJs around 66 MS and subgiant stars in M67 (53 stars if we only consider single stars). This gives a frequency of HJs of $4.5_{-2.5}^{+4.5} \%$ and $5.6_{-2.6}^{+5.4} \%$. These results also agree with the HJs frequency $\left(5.5_{-2.5}^{+5.5} \%\right)$ obtained by a Monte Carlo analysis in our parallel work (Brucalassi et al. 2016). Our values are higher than those derived from the RV surveys around FGK stars $(1.2 \% \pm 0.38$ of HJs Wright et al. 2012). The comparison is

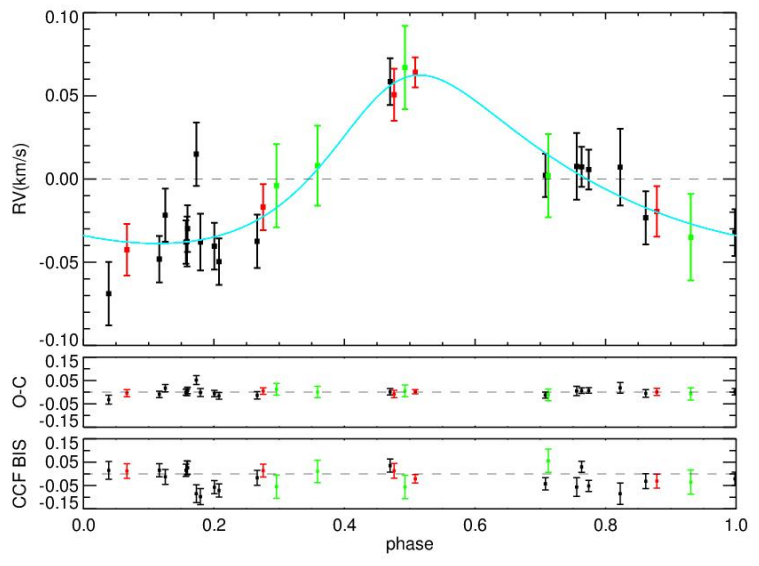

Fig. 3. Phased RV measurements and Keplerian best fit, best-fit residuals, and bisector variation for YBP1514. Same symbols as in Fig. 2.

even more striking when considering that the Kepler ${ }^{1}$ statistics of HJs is lower, around $0.4 \%$ (Howard et al. 2011). However, the comparison between different samples and between simulations and observations is not trivial. The analysis of the Kepler and the RV surveys for instance use different selection criteria (radii vs. masses) and different intervals of orbital periods. Dawson \& Murray-Clay (2013) showed that the discrepancy might be partially due to the different metallicity of the samples. Another effect to take into account is in the definition of the comparison samples. RV surveys are performed on pre-selected samples that have been corrected for the presence of binaries, while the Kepler statistics (and most of the simulations) refer to all FGK stars in the Cygnus field, without any previous selection for binaries. For M67 our survey sample was heavily pre-selected with the aim to eliminate all known and suspected binaries in advance. We therefore expect that when we compare our results on the whole sample (3/66 or $\left.4.5_{-2.5}^{+4.5} \%\right)$ with the Kepler statistics $(0.4 \%)$, an upper limit of the planet frequency will be provided, while the comparison of the frequency of the single-star sample $\left(3 / 53\right.$ or $5.6_{-2.6}^{+5.4 \%)}$ ) is expected to compare well with the $1.2 \%$ of the radial velocity surveys because they have gone through a similar selection process. Finally, based on a $\mathrm{HJ}$ occurrance rate of $1.2 \%$ like for field stars, one or two additional HJs may exist in M67 with a non-negligible probability of $5 \%$.

For several years, the lack of detected planets in OCs was in contrast with the field results, but the recent discoveries (Malavolta et al. 2016; Brucalassi et al. 2014; Meibom et al. 2013; Quinn et al. 2014, 2012) have completely changed the situation. These results are difficult to reconcile with the early survey of Paulson et al. (2004), who did not detect any HJs around 94 G-M stars of the Hyades cluster. Since the extrapolation from non-detection to non-existence of HJs critically depends on several assumptions such as stellar noise and real measurement errors, constraints on the allocated time, number of observations per star, sampling and planet mass, we cannot state at present whether the discrepancy is real until larger surveys are performed. If we were to add the results of the RV surveys in the OCs M67, Hyades, and Praesepe, we would determine a rate of 6 out of $240 \mathrm{HJ}$ per surveyed stars (including some binaries), which is a high percentage when compared to 10 out of $836 \mathrm{HJ}$ per surveyed stars in the field sample of Wright et al. (2012). We can conclude that, contrary to early reports, the frequency of HJs discovered in the three OCs subject of recent RV surveys is higher than amongst the field stars. To explain the high

1 http://kepler.nasa.gov/ 
frequency of HJs in M67, Hyades, and Praesepe, we may argue that the frequency of HJs depends on stellar metallicity, mass, or on dynamical history, and therefore environment. The dependence of planet frequency on stellar metallicity is complex: even if established very early (Johnson et al. 2010; Udry \& Santos 2007; Fischer \& Valenti 2005; Santos et al. 2004), a real correlation seems to be present only for Jupiters around MS stars, while it does not hold for giant planets around evolved stars (Pasquini et al. 2007) or for low-mass planets (Mayor et al. 2011). Both Hyades and Praesepe are metal rich (Pace et al. 2008; DutraFerreira et al. 2016), and this may explain the higher frequency of HJs in these clusters, but this is not the case of M67, which has a well-established solar metallicity and abundance pattern (Randich et al. 2005). The hypothesis that the high frequency of HJs in M67 or in OCs in general originates from the higher mass of the host star can be also excluded: the stars hosting HJs in M67 all have masses around one solar mass, which is very similar to the masses of the HJ hosts discovered in the field. A similar argument holds for the stars hosting HJs in Praesepe and in the Hyades.

Finally, environment is left as the most suitable option to explain the HJs excess. It has been suggested that dense birthenvironments such as stellar clusters can have a significant effect on the planet formation process and the resulting orbital properties of single planets or planetary systems. Close stellar fly-by or binary companions can alter the structure of any planetary system and may also trigger subsequent planet-planet scattering over very long timescales (Davies et al. 2014; Malmberg et al. 2011). This leads to the ejection of some planets, but it also seems to favour the conditions for the formation of HJs (Shara et al. 2016). As predicted by such mechanisms, M67 HJs show orbits with non-zero eccentricity, which is also true for the HJ found in the Hyades. The importance of the encounters is primarly determined by the local stellar density, the binary fraction, the collisional cross-section of the planetary system, and the timescale on which the planet is exposed to external perturbations. Malmberg et al. (2011) produced simulations for a cluster of 700 stars and an initial half-mass radius of 0.38 parsec, showing that a non-negligible number of stars spend long enough as a binary system and also that the majority of the stars is affected by at least one fly-by. M67 has more than 1400 stars at present, it is dominated by a high fraction of binaries (Davenport \& Sandquist 2010) after loosing at least three quarters of its original stellar mass, and has suffered mass segregation. Shara et al. (2016) have recently completed $N$-body simulations for a case similar to the one of M67, but with only a $10 \%$ of binaries, finding that HJs can be produced in $0.4 \%$ of cluster planetary systems when only considering initial fly-by encounters. This fraction is smaller than what we find in M67, and the influence of other migration mechanisms probably needs to be considered as well to explain our results. However, the same authors acknowledged that a higher fraction of binaries will strongly enhance the probability of $\mathrm{HJ}$ formation and therefore their frequency. Given that the binary fraction of M67 stars is currently very high (Pasquini et al. 2012; Mathieu et al. 1990) and that models show that it must also have been high at the origin (Hurley et al. 2005), the high fraction of M67 HJs seems to qualitatively agree with the $N$-body simulations. The same simulations predict that after 5 Gyr the percentage of stars hosting HJs retained by the cluster is substantially higher than the percentage of stars not hosting HJs. Two factors can contribute to enhance HJ planet formation: the capability of producing HJs, and the capability of the cluster to retain stars hosting HJs. The interaction takes part well within the first Gyr of the cluster lifetime, so that the stars with HJs do not require to still have a stellar companion at the age of M67. Geller et al. (2015) reported no evidence for nearby companions at the present epoch. Finally, considering that about one of ten HJs produces a transit, we suggest to carefully examine the Kepler/K2 observations (Howell et al. 2014) for any transit.

Acknowledgements. L.P. acknowledges the Visiting Researcher program of the CNPq Brazilian Agency, at the Fed. Univ. of Rio Grande do Norte, Brazil. RPS thanks ESO DGDF, the HET project, the PNPS and PNP of INSU CNRS for allocating the observations. MTR received support from PFB06 CATA (CONICYT).

\section{References}

Baranne, A., Queloz, D., Mayor, M., et al. 1996, A\&AS, 119, 373 Bouchy, F., \& Sophie Team. 2006, in Tenth Anniversary of 51 Peg-b, eds. L. Arnold, F. Bouchy, \& C. Moutou, 319

Brucalassi, A., Pasquini, L., Saglia, R., et al. 2014, A\&A, 561, L9 (Paper II) Cappetta, M., Saglia, R. P., Birkby, J. L., et al. 2012, MNRAS, 427, 1877 Davenport, J. R. A., \& Sandquist, E. L. 2010, ApJ, 711, 559

Davies, M. B., Adams, F. C., Armitage, P., et al. 2014, Protostars and Planets VI,

H. Beuther, R. S. Klessen, C. P. Dullemond, \& T. Hennings (Tucson: Univ. Arizona Press), 787

Dawson, R. I., \& Murray-Clay, R. A. 2013, ApJ, 767, L24

Dutra-Ferreira, L., Pasquini, L., Smiljanic, R., et al. 2016, A\&A, 585, A75

Fischer, D. A., \& Valenti, J. 2005, ApJ, 622, 1102

Geller, A. M., Latham, D. W., \& Mathieu, R. D. 2015, AJ, 150, 97

Girardi, L., Bressan, A., Bertelli, G., \& Chiosi, C. 2000, A\&AS, 141, 371

Goldreich, P., \& Tremaine, S. 1980, ApJ, 241, 425

Horne, J. H., \& Baliunas, S. L. 1986, ApJ, 302, 757

Howard, A. W., Marcy, G. W., Bryson, S. T., et al. 2011, ApJ, 730, 10

Howell, S. B., Sobeck, C., Haas, M., et al. 2014, PASP, 126, 398

Hurley, J. R., Pols, O. R., Aarseth, S. J., \& Tout, C. A. 2005, MNRAS, 363, 293 Johnson, J. A., Aller, K. M., Howard, A. W., \& Crepp, J. R. 2010, PASP, 122, 905

Lin, D. N. C., \& Ida, S. 1997, ApJ, 477, 781

Lin, D. N. C., \& Papaloizou, J. 1986, ApJ, 309, 846

Lovis, C., \& Mayor, M. 2007, A\&A, 472, 657

Malavolta, L., Nascimbeni, V., Piotto, G., et al. 2016, A\&A, 588, A118

Malmberg, D., Davies, M. B., \& Heggie, D. C. 2011, MNRAS, 411, 859

Mathieu, R. D., Latham, D. W., \& Griffin, R. F. 1990, AJ, 100, 1859

Mayor, M., Pepe, F., Queloz, D., et al. 2003, The Messenger, 114, 20

Mayor, M., Marmier, M., Lovis, C., et al. 2011, A\&A, submitted [arXiv: 1109.2497]

Meibom, S., Torres, G., Fressin, F., et al. 2013, Nature, 499, 55

Önehag, A., Korn, A., Gustafsson, B., et al. 2011, A\&A, 528, A85

Pace, G., Pasquini, L., \& François, P. 2008, A\&A, 489, 403

Pace, G., Castro, M., Meléndez, J., et al. 2012, A\&A, 541, A150

Pasquini, L., \& Pallavicini, R. 1991, A\&A, 251, 199

Pasquini, L., Döllinger, M. P., Weiss, A., et al. 2007, A\&A, 473, 979

Pasquini, L., Biazzo, K., Bonifacio, P., et al. 2008, A\&A, 489, 677

Pasquini, L., Brucalassi, A., Ruiz, M. T., et al. 2012, A\&A, 545, A139

Paulson, D. B., Saar, S. H., Cochran, W. D., \& Henry, G. W. 2004, AJ, 127, 1644

Pepe, F., Mayor, M., Galland, F., et al. 2002, A\&A, 388, 632

Pietrinferni, A., Cassisi, S., Salaris, M., \& Castelli, F. 2004, ApJ, 612, 168

Queloz, D., Henry, G. W., Sivan, J. P., et al. 2001, A\&A, 379, 279

Quinn, S. N., White, R. J., Latham, D. W., et al. 2012, ApJ, 756, L33

Quinn, S. N., White, R. J., Latham, D. W., et al. 2014, ApJ, 787, 27

Randich, S., Bragaglia, A., Pastori, L., et al. 2005, The Messenger, 121, 18

Rasio, F. A., \& Ford, E. B. 1996, Science, 274, 954

Sanders, W. L. 1977, A\&AS, 27, 89

Santerne, A., Díaz, R. F., Moutou, C., et al. 2012, A\&A, 545, A76

Santos, N. C., Israelian, G., \& Mayor, M. 2004, A\&A, 415, 1153

Scargle, J. D. 1982, ApJ, 263, 835

Shara, M. M., Hurley, J. R., \& Mardling, R. A. 2016, ApJ, 816, 59

Taylor, B. J. 2007, AJ, 133, 370

Tull, R. G. 1998, in SPIE Conf. Ser. 3355, ed. S. D'Odorico, 387

Udry, S., \& Santos, N. C. 2007, ARA\&A, 45, 397

Vereshchagin, S. V., Chupina, N. V., Sariya, D. P., et al. 2014, New Astron., 31, 43

Ward, W. R. 1997, Icarus, 126, 261

Wright, J. T., \& Howard, A. W. 2009, ApJS, 182, 205

Wright, J. T., Marcy, G. W., Howard, A. W., et al. 2012, ApJ, 753, 160

Yadav, R. K. S., Bedin, L. R., Piotto, G., et al. 2008, A\&A, 484, 609 
A. Brucalassi et al.: Search for giant planets in M67. III.

\section{Appendix A: Additional figures and tables}

RV values
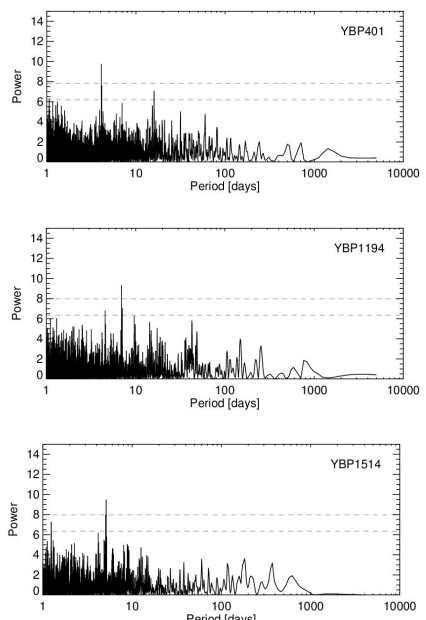

Residuals
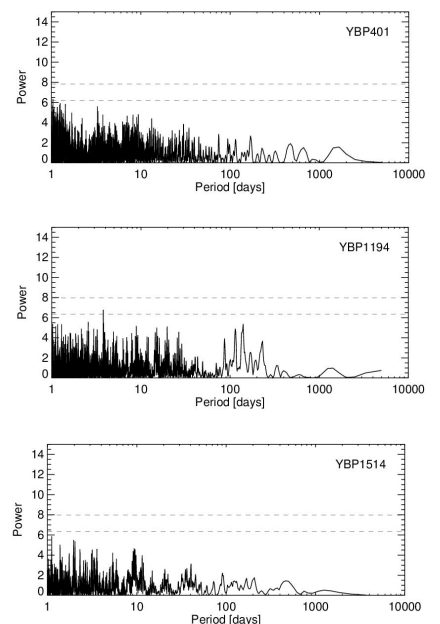

BIS span
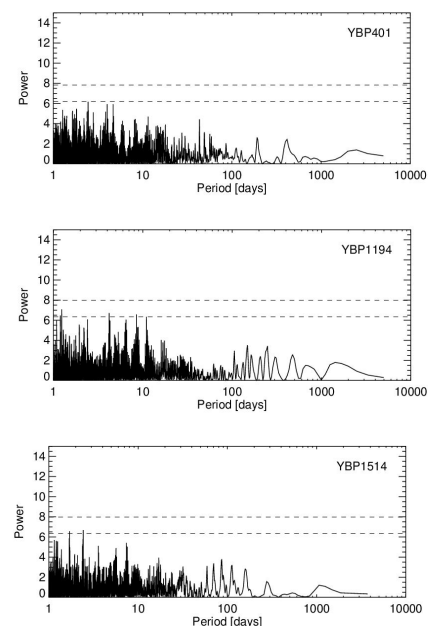

FWHM
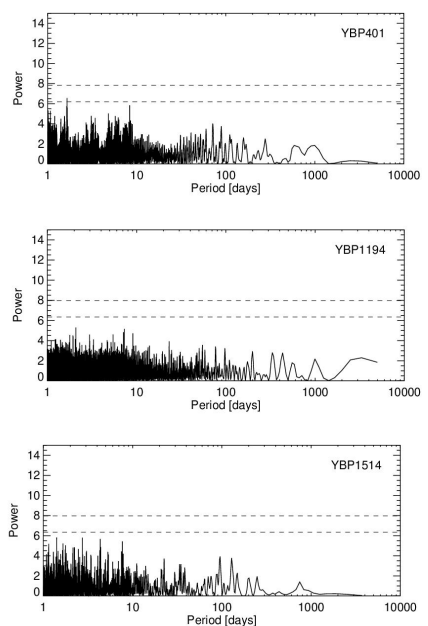

Fig. A.1. Top: Lomb-Scargle periodogram of the RV measurements, residuals, bisector span, and FWHM for YBP401. Central: same plots for YBP1194. Bottom: same plots for YBP1514. The dashed lines correspond to 5\% and 1\% false-alarm probabilities, calculated according to Horne \& Baliunas (1986) and white noise simulations.
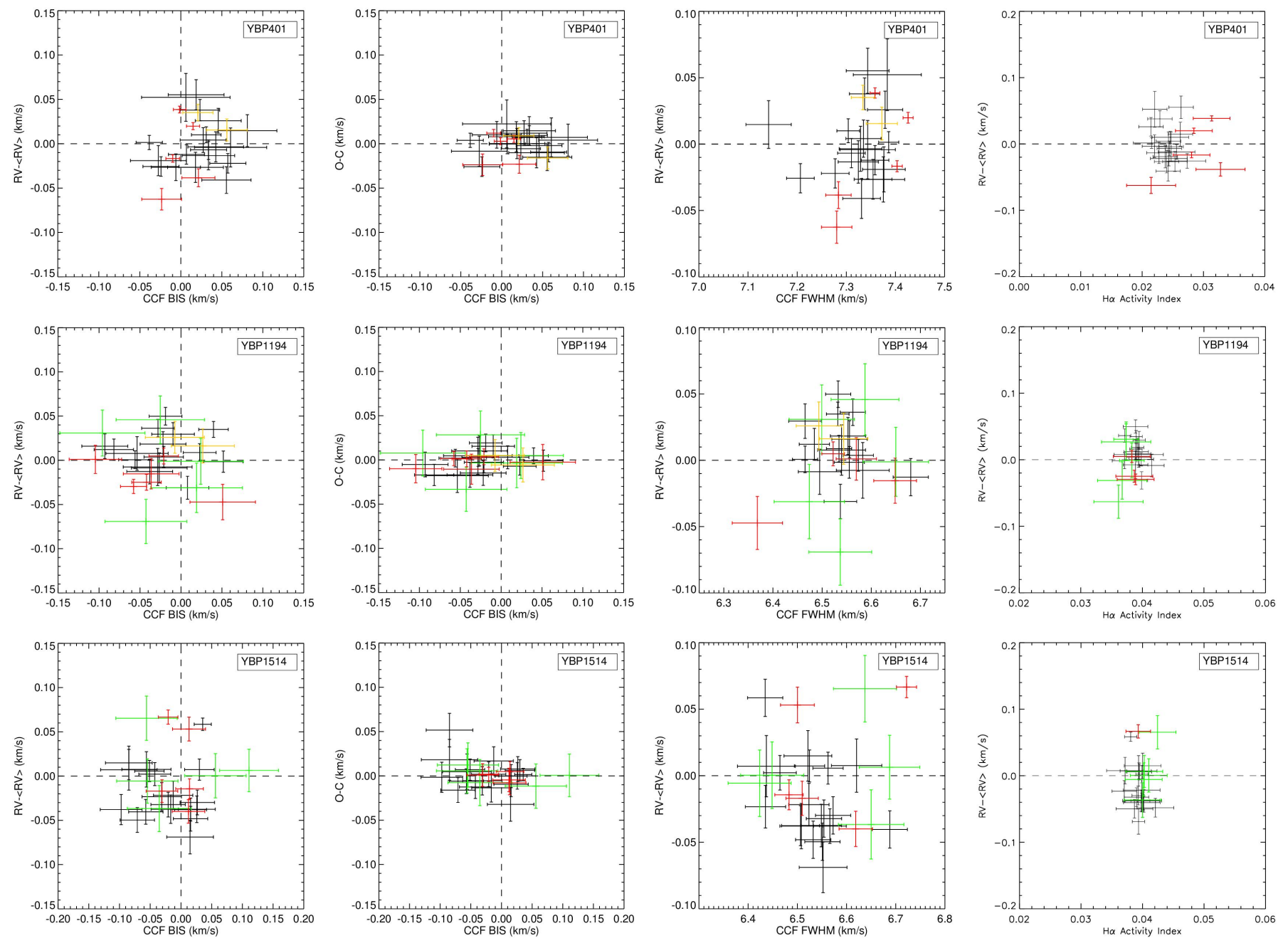

Fig. A.2. Top: RV measurements versus bisector span, residuals versus bisector span, RV measurements versus CCF FWHM and RV measurements versus $\mathrm{H} \alpha$ activity indicator for YBP401. The $\mathrm{H} \alpha$ activity indicator is computed as the area below the core of $\mathrm{H} \alpha$ line with respect to the continuum. CCF FWHM values are calculated by subtracting the respective instrumental FWHM in quadrature. Same symbols as in Fig. 2. Middle: the same plots for YBP1194. Bottom: the same plots for YBP1514. 
Table A.1. RV measurements, RV uncertainties, bisector span, and ratio of the $\mathrm{H}_{\alpha}$ core with respect to the continuum (see Pasquini \& Pallavicini 1991) for YBP401.

\begin{tabular}{|c|c|c|c|c|c|}
\hline $\begin{array}{l}\text { BJD } \\
(-2450000)\end{array}$ & $\begin{array}{r}\mathrm{RV} \\
\left(\mathrm{km} \mathrm{s}^{-1}\right) \\
\end{array}$ & $\begin{array}{r}\sigma_{\mathrm{obs}} \\
\left(\mathrm{km} \mathrm{s}^{-1}\right) \\
\end{array}$ & $\begin{array}{r}\text { BIS span } \\
\left(\mathrm{km} \mathrm{s}^{-1}\right) \\
\end{array}$ & $\mathrm{H}_{\alpha}$ ratio & instrument \\
\hline 4491.3462 & 33.2165 & 0.0089 & -0.0013333 & 0.03133 & SOPHIE \\
\hline 4855.6494 & 33.1522 & 0.0110 & -0.0241850 & 0.02735 & HARPS \\
\hline 4859.5234 & 33.1978 & 0.0089 & 0.0150000 & 0.02843 & SOPHIE \\
\hline 4861.8052 & 33.1559 & 0.0110 & 0.0573989 & 0.02435 & HARPS \\
\hline 5190.8584 & 33.1793 & 0.0080 & -0.0384314 & 0.02154 & HARPS \\
\hline 5627.7314 & 33.2302 & 0.0270 & 0.0061686 & 0.02206 & HARPS \\
\hline 5946.8267 & 33.1927 & 0.0180 & 0.0812266 & 0.02624 & HARPS \\
\hline 5967.6030 & 33.1589 & 0.0170 & -0.0273837 & 0.02377 & HARPS \\
\hline 5978.5732 & 33.2331 & 0.0170 & 0.0187173 & 0.02635 & HARPS \\
\hline 5985.5850 & 33.1612 & 0.0090 & -0.0098333 & 0.02806 & SOPHIE \\
\hline 6245.8511 & 33.1369 & 0.0150 & 0.0556374 & 0.02424 & HARPS \\
\hline 6269.7925 & 33.1744 & 0.0170 & 0.0426396 & 0.02201 & HARPS \\
\hline 6302.7832 & 33.1737 & 0.0220 & 0.0607550 & 0.02471 & HARPS \\
\hline 6308.7578 & 33.1658 & 0.0110 & 0.0069258 & 0.02561 & HARPS \\
\hline 6323.7534 & 33.1513 & 0.0170 & 0.0179823 & 0.02399 & HARPS \\
\hline 6333.7314 & 33.2038 & 0.0140 & 0.0456952 & 0.02144 & HARPS \\
\hline 6682.6787 & 33.1879 & 0.0090 & 0.0310414 & 0.02459 & HARPS \\
\hline 6694.5210 & 33.1934 & 0.0124 & 0.0562494 & - & HARPS-N \\
\hline 6697.4912 & 33.2130 & 0.0092 & 0.0208414 & - & HARPS-N \\
\hline 6715.7002 & 33.1711 & 0.0110 & 0.0338823 & 0.02443 & HARPS \\
\hline 6719.6357 & 33.1821 & 0.0140 & 0.0273266 & 0.02430 & HARPS \\
\hline 6720.4028 & 33.1154 & 0.0145 & -0.0233333 & 0.02147 & SOPHIE \\
\hline 6721.5381 & 33.1395 & 0.0129 & 0.0215000 & 0.03277 & SOPHIE \\
\hline 6977.8101 & 33.1514 & 0.0150 & -0.0060115 & 0.02535 & HARPS \\
\hline 6978.8369 & 33.1645 & 0.0160 & 0.0337306 & 0.02274 & HARPS \\
\hline 6983.8188 & 33.2158 & 0.0120 & 0.0234168 & 0.02289 & HARPS \\
\hline
\end{tabular}

Notes. All the RV data points are corrected to the zero point of HARPS.

Table A.2. Orbital parameters of the planetary candidates using a simple MCMC analysis to fit Keplerian orbits to the RV data.

\begin{tabular}{lrrr}
\hline \hline Parameters & YBP401 & YBP1194 & YBP1514 \\
\hline$P$ [days $]$ & $4.0873_{-0.0002}^{+0.0003}$ & $6.959_{-0.001}^{+0.001}$ & $5.1189_{-0.0007}^{+0.0008}$ \\
$T[\mathrm{JD}]$ & $255974.23_{-0.49}^{+0.49}$ & $2455290.0_{-0.3}^{+0.4}$ & $245986.34_{-0.20}^{+0.28}$ \\
$e$ & $0.141_{-0.113}^{+0.112}$ & $0.294_{-0.056}^{+0.077}$ & $0.332_{-0.127}^{+0.133}$ \\
$\omega[\mathrm{deg}]$ & $-31.69_{-54.0}^{+43.0}$ & $99.14_{-16.0}^{+16.0}$ & $-34.76_{-14.26}^{+17.35}$ \\
$\sqrt{e} \sin \omega$ & $-0.197_{-0.166}^{+0.152}$ & $0.535_{-0.064}^{+0.061}$ & $-0.329_{-0.136}^{+0.134}$ \\
$\sqrt{e} \cos \omega$ & $0.319_{-0.242}^{+0.104}$ & $-0.086_{-0.111}^{+0.186}$ & $0.474_{-0.086}^{+0.092}$ \\
$K\left[\mathrm{~m} \mathrm{~s}^{-1}\right]$ & $48.911_{-6.0}^{+5.0}$ & $35.607_{-4.0}^{+2.2}$ & $50.97_{-3.0}^{+3.0}$ \\
$m \sin i\left[M_{\text {Jup }}\right]$ & $0.41_{-0.04}^{+0.06}$ & $0.32_{-0.2}^{+0.3}$ & $0.42_{-0.02}^{+0.03}$ \\
$\gamma\left[\mathrm{km} \mathrm{s}^{-1}\right]$ & $33.172_{-0.004}^{+0.003}$ & $34.185_{-0.002}^{+0.002}$ & $34.058_{-0.003}^{+0.003}$ \\
\hline
\end{tabular}

Notes. We considered as free parameters the orbital period $P$, the time of transit $T_{\mathrm{c}}$, the radial velocity semi-amplitude $K$, the centre-of-mass velocity $\gamma$, and the orthogonal quantities $\sqrt{e} \cos \omega$ and $\sqrt{e} \sin \omega$, where $e$ is the eccentricity and $\omega$ is the argument of periastron. We quote the mode of the resulting parameter distributions as the final value and the $68.3 \%$ interval with equal probability density at the $\pm 1 \sigma$ bound to derive the uncertainty. $T$ : time at periastron passage, $K$ : semi-amplitude of the RV curve, $m \sin i$ : planetary minimum mass. 